\title{
LAS TUTORÍAS DE ESCRITURA ACADÉMICA PRESENCIALES Y VIRTUALES: ¿ QUUÉ PODEMOS APRENDER SOBRE SUS ESTRATEGIAS DIDÁCTICAS?
}

\author{
Face-to-Face and Virtual Academic Writing Tutoring Sessions: What Can Be \\ LEARNT FROM THEIR DidACTIC STRATEgies? \\ LES TUTORATS D'ÉCRITURE ACADÉMIQUE PRÉSENTIELS ET VIRTUELS : QU'EST-CE QUE \\ NOUS POUVONS APPRENDRE SUR SES STRATÉGIES DIDACTIQUES?
}

\author{
Juan Antonio Núñez-Cortés \\ Profesor Ayudante Doctor, \\ Universidad Autónoma de Madrid, \\ Madrid, España. \\ juanantonio.nunnez@uam.es \\ https://orcid. \\ org/0000-0003-0494-3850

\section{María Constanza Errázuriz \\ Profesora Asociada, Pontificia \\ Universidad Católica de Chile, \\ Villarrica, Chile. \\ cerrazuc@uc.cl \\ https://orcid.} \\ org/0000-0001-7976-9397
}

\section{Adrián Neubauer Esteban}

Contratado predoctoral, Universidad

Autónoma de Madrid, Madrid,

España.

adrian.neubaver@uam.es

https://orcid.

org/0000-0002-6774-1538

\section{Claudia Parada \\ Profesora Asistente, Pontificia \\ Universidad Católica de Chile, \\ Villarrica, Chile. \\ cparadao@uc.cl \\ https://orcid. \\ org/0000-0002-4286-8268}

\begin{abstract}
RESUMEN
En el contexto hispánico, han surgido los centros de escritura y las tutorías, los cuales se han debido adaptar a la educación a distancia, por causa de la emergencia sanitaria de la Covid-19. No obstante, aún hay pocas evidencias con respecto a cómo funcionan las tutorías en esa modalidad de educación. A partir de lo anterior, este trabajo tuvo como objetivo analizar las estrategias didácticas que utilizaron dos tutoras pares durante las tutorías de escritura presenciales que ofrecieron antes de la pandemia y las virtuales, realizadas durante la pandemia, luego de un programa específico de formación. Para ello, mediante una investigación cuantitativa-cualitativa, se realizó un análisis de contenido, por medio del software MAXQDA, de las transcripciones de 16 tutorías ( 8 presenciales y 8 virtuales). Los resultados dan cuenta de una prevalencia general de estrategias cognitivas, sobre todo la pregunta, junto con instructivas, como la sugerencia y la explicación. No obstante, se perciben diferencias en los tipos de tutorías y se confirma una tendencia a centrarse más en el estudiante y en el proceso en las tutorías virtuales. Estos resultados sugieren que las tutorías virtuales son una alternativa útil en el acompañamiento del proceso de escritura a estudiantes universitarios.
\end{abstract}

Palabras clave: centros de escritura; COVID-19; escritura académica; estrategias didácticas; tutorías presenciales; tutorías virtuales.

\section{Abstract}

In Spanish-speaking settings, newly-emerged writing tutoring centers have seen the need to adapt to emergency remote education due to the health emergency brought about by COVID-19. However, there is lack of evidence regarding how

Recibido : 2021-02-28 / Aceptado : 2021-06-11 / Publicado : 2021-09-11

https://doi.org/10.17533/udea.ikala.v26n3a10

Edición especial Elrol de la tecnologia en la enseñanza y aprendizaje de lenguas durante la crisis generadapor lapandemia de COVID-19. Editores: Marta González-Lloret, University of Hawai'i at Mānoa, EE.uU; Laia Canals, Universitat Oberta de Catalunya, España; Jorge Pineda, Universidad de Antioquia, Colombia.

(C) 2021 Universidad de Antioquia. Este es un artículo de acceso abierto, distribuido según los términos de la licencia de Creative Commons BY-NC-SA 4.0 Internacional. 
writing tutoring actually works under this education modality. Drawing on the above, this article aimed to analyse the didactic strategies used by two peer tutors in both face-to-face writing tutoring sessions that occurred before the pandemic and online sessions taking place during the pandemic, after having undergone a specific training program. Data were collected through a quantitative-qualitative approach, and the MAXQDA software was used to do a content analysis of 16 tutoring session ( 8 face-to-face and 8 online) transcriptions. Findings show a prevalence of cognitive approaches, mainly questioning; along with instructional approaches, such as suggestion and explanation. Nonetheless, differences are perceived between the various types of tutoring sessions, along with a trend toward a more student-centered approach in online tutoring sessions. These results suggest online tutoring sessions are a useful alternative when accompanying the writing process in university students.

Keywords: Writing centers; COVID-19; academic writing; didactic strategies; face-to-face tutoring sessions; online tutoring sessions.

\section{RÉSUMÉ}

Dans le contexte hispanique, des centres d'écriture et leurs tutorats émergents ont dû s'adapter à l'enseignement à distance, en raison de l'urgence sanitaire du COvid-19. Cependant, il n'y pas beaucoup d'évidence sur le fonctionnement des sessions de tutorat dans ce mode d'éducation. Sur la base de ce qui précède, ce travail visait à analyser les stratégies didactiques utilisées par deux pairs tuteurs dans des tutoriels d'écriture en présentiel avant la pandémie et virtuels pendant la pandémie, après un modèle de formation spécifique. Pour ce faire, à travers une recherche quantitative-qualitative, une analyse de contenu a été réalisée, à l'aide du logiciel MAXQDA, des transcriptions de 16 tutoriels ( 8 en présentiel et 8 virtuels). Les résultats montrent une prévalence générale des stratégies cognitives, en particulier la question, ainsi que des stratégies instructives, telles que la suggestion et l'explication. Cependant, des différences sont perçues dans les types de tutoriels, ce qui confirme une tendance à se concentrer davantage sur l'étudiant et le processus dans les tutoriels virtuels. Ces résultats suggèrent que les tutorats virtuels sont une alternative utile pour accompagner le processus d'écriture des étudiants universitaires.

Mots-clés : centres d'écriture ; COVID-19 ; écriture académique ; stratégies d'enseignement ; tutoriels présentiels ; tutoriels virtuels. 


\section{Introducción}

Frente a los obstáculos y desafíos de escritura que encaran los estudiantes universitarios al ingresar a la educación superior, son diferentes las iniciativas que se pueden implementar para abordarlos. Así, se despliegan iniciativas puntuales, remediales o de nivelación, como cursos de comunicación escrita al inicio de la carrera, talleres sobre géneros discursivos académicos o recursos digitales que orientan acerca de aspectos formales de la lengua, las fases del proceso de escritura, los géneros académicos o las convenciones específicas de las disciplinas. También se ofrecen soluciones más sistemáticas y transversales, como las asesorías y el trabajo conjunto entre profesores especialistas en la enseñanza de la escritura y docentes de las asignaturas, así como propuestas de escritura insertas en el currículo o desarrolladas en las disciplinas de los programas de formación y las tutorías de los centros de escritura.

Si bien estas propuestas más sistemáticas, en varios casos, se han llevado a cabo inicialmente para elevar la calidad de la formación profesional (Thaiss et al., 2012), asimismo se han consolidado como dispositivos que promueven la equidad y la inclusión, pues responden a las barreras que enfrenta el estudiantado al tratar de adaptarse a las prácticas de lectura y escritura de las comunidades académicas (Natale y Stagnaro, 2017). Estas iniciativas han sido particularmente relevantes en el contexto iberoamericano, donde la admisión a la educación superior ha tenido un notable crecimiento, debido a políticas públicas de equidad y democratización de la educación, por lo que se ha recibido una mayor cantidad de estudiantes tradicionalmente excluidos o desaventajados por razones socioeconómicas, étnicas, territoriales o de género (Ezcurra, 2013).

Como lo mencionamos anteriormente, dentro de las iniciativas más sistemáticas se inscriben las tutorias de escritura, que atienden a los estudiantes en el marco de un centro o programa de escritura, y también en proyectos de innovación docente (Núñez y Errázuriz, 2020). Estas tutorías corresponden a instancias de atención personalizada o en pequeños grupos de estudiantes, cuya naturaleza en general contribuye a imprimir una mayor calidad y equidad en la formación. Entre los motivos que fundamentan ofrecer estas instancias a la medida para los estudiantes destacan las características personales de cada uno de ellos; la diversidad de sus demandas, dificultades e intereses, así como la falta de formación de muchos profesores en la enseñanza de la escritura en las disciplinas que imparten (Núñez, 2020).

Sin embargo, aún contamos con poca información respecto de lo que ocurre en la instancia de interacción de tutoría en el ámbito hispánico, especialmente en línea, dado que casi la totalidad de las investigaciones disponibles han sido realizadas en el contexto anglosajón. Es más, la emergencia sanitaria de pandemia provocada por la COVID-19 ha ahondado la brecha de desigualdad en educación, debido a la dificultad de acceso a la enseñanza en línea, la deserción estudiantil y la falta de formación de los docentes sobre la enseñanza a distancia (Unesco, 2020). Asimismo, dado que en la mayor parte de los centros de escritura las tutorías son presenciales, estos se han visto en la necesidad de reformular sus prácticas y adaptar las tutorías a la modalidad virtual.

Por ello, el objetivo general de esta investigación fue analizar las estrategias didácticas que utilizaron dos tutoras pares en las tutorías de escritura presenciales antes de la pandemia y virtuales durante la pandemia, luego de un modelo específico de formación. Así, este trabajo pretende dar cuenta de las dinámicas que surgen en las tutorías y que se pueden tener en consideración en la futura formación sobre didáctica de la escritura y, en concreto, sobre la retroalimentación de textos de tutores de escritura y docentes de diferentes niveles académicos.

\section{Marco teórico}

En esta sección, se presenta el concepto de tutoría de escritura y se atiende a los fundamentos pedagógicos de las tutorías y sus características, 
así como a sus aspectos positivos. Por último, se exponen las diferentes estrategias didácticas que se pueden llevar a cabo en las tutorías

\section{Conceptualización de las tutorías de escritura}

Las tutorías de escritura, cuyos tutores son estudiantes, son consideradas como tutorías entre iguales o pares. En ellas, tutores pares con mayor experiencia en la carrera y en tareas de expresión escrita monitorean el proceso de escritura de un texto de estudiantes menos preparados (Alzate y Peña, 2010). Por tanto, por lo general, los tutores son de cursos y edades superiores.

Además de su mayor experiencia académica, los tutores estudiantes cuentan con una formación explícita para realizar el rol de tutores de escritura en el marco de un centro de escritura, o de programas o proyectos de innovación enfocados en el desarrollo de la escritura. Así, el vínculo que se construye entre el tutor y el tutorado es asimétrico; no obstante, es más simétrico que la relación entre profesor y alumno. Por ende, esta relación promueve un aprendizaje mutuo, debido a que no se califica el texto trabajado y los participantes son alumnos universitarios que están pasando por experiencias similares (Chois et al., 2017).

En consecuencia, la tutoría de escritura es una acción pedagógica, en la que un tutor formado interactúa oralmente con un estudiante y acompaña su proceso de escritura de un texto académico o disciplinar. Esto, con el propósito de fomentar la reflexión sobre el texto y el tema abordado, la metacognición acerca del proceso de escritura y las estrategias aplicadas y, así, promover la mejora del estudiante como escritor (Núñez, 2020). De igual modo, la tutoría en línea es concebida de manera similar, pero con la particularidad de que este trabajo es mediado por la tecnología, a través de diversas plataformas o recursos, tanto de forma sincrónica como asincrónica (Hewett, 2006; Skagen et al., 2009).

Respecto del rol de tutor, cabe destacar la idea de North (1982) de que este no es un editor ni corrector de textos. En cambio, sí está entre sus funciones fomentar que el estudiante desarrolle su sentido de autoría, formular preguntas a partir del texto (Alzate y Peña, 2010) y apoyar el aprendizaje sobre la escritura (Cooper, 1994). Así, el tutor no debe imponer su estilo de escritura o presentar el suyo como el único adecuado; por el contrario, debe respetar el estilo ajeno y apoyarse en la conformación de la identidad del estudiante como escritor. Por ende, en la tutoría, el tutor llevará a cabo diversos roles, por ejemplo, de lector, comentarista, aprendiz, consejero, colaborador, entre otros (Ryan y Zimmerelli, 2006).

\section{Fundamentos pedagógicos y características de las tutorías de escritura}

Las tutorías de escritura se basan inicialmente en las teorías constructivista y sociocultural de Piaget (1970) y Vygotsky (1978), respectivamente. En este sentido, serían, de acuerdo con Monty (2013), un espacio en donde se favorece la "zona de desarrollo próximo” —entendida como el espacio entre lo que un individuo es capaz de hacer por sí mismo y lo que puedo aprender gracias a la ayuda de otro-, y a través de la interacción oral entre estudiante y tutor, el primero realiza una tarea que no habría podido lograr por sí mismo (Castellà y Aliagas, 2016). De ahí que durante la tutoría se promueva, en palabras de Scardamalia y Bereiter (1992), una transformación del conocimiento mientras se escribe y se reflexiona, y no una simple reproducción de este. Es decir, el conocimiento es reflexionado y mediado por el tutorado y el tutor, a través del proceso escritura y la interacción sobre este.

En cuanto a las experiencias exitosas de tutorías de escritura tanto en España como en Latinoamérica, se han constatado algunos de los beneficios que aportan, dado que: 1) promueven la integración, en la comunidad académica, de estudiantes de los primeros años de la carrera, de quienes presentan dificultades en escritura y de aquellos que tienen diferentes necesidades; 2) brindan la posibilidad de conocer géneros discursivos académicos y dialogar sobre ellos; 3 ) elevan la motivación hacia la mejora 
de la escritura, las actitudes positivas hacia esta, promueven el autoconcepto como escritores, la autoeficacia y, por tanto, el desempeño académico; 4) ofrecen una experiencia de aprendizaje valiosa para ambas partes, pues los tutores toman conciencia sobre su propio aprendizaje al activar procesos de metacognición; 5) fomentan valores de solidaridad y socialización, y aportan al desarrollo personal, académico y profesional de los tutores, 6) flexibilizan el tiempo y el espacio, en el caso de la tutoría virtual (Alzate y Peña, 2010; Bach y Montané, 2016; Castellà y Aliagas, 2016; Chois et al., 2017; Errázuriz, 2016, 2017; Holtz, 2014; Jones et al., 2006; Kirchhoff, 2016; Skagen et al., 2009; Yergeau et al., 2019), y 7) suponen un reconocimiento académico o económico para los tutores.

En relación con el desarrollo de las tutorías, hay diferentes maneras de llevarlas a cabo, por lo que los estilos de los tutores pueden ser diversos (Dinitz y Kiedaisch, 2003). De hecho, la pertenencia del tutor a la misma disciplina del estudiante se ha considerado especialmente importante (Cromley y Azevedo, 2005). En este sentido, se han reconocido diferentes tipos de tutorías (García-Arroyo y Quintana, 2016; Roldán y Arenas, 2016) y, en general, se pueden identificar dos clases contrapuestas: las primeras se focalizan en el estudiante y en el proceso, por lo que es el tutorado quien lee el texto, formula dudas de escritura y recoge las recomendaciones del tutor. En cambio, en las tutorías enfocadas en el texto o producto, y el tutor, es este último quien protagoniza la sesión y lee el texto, identifica los errores y emite preguntas que el estudiante responde. No obstante, si bien estos son dos modelos dicotómicos, entre ambos extremos pueden presentarse diversos tipos de tutorías híbridas, que internalizan ambos modelos, pero en las que predomina uno u otro según las circunstancias, el contexto y la experticia del tutor.

A nuestro juicio, sería recomendable promover un modelo de tutorías de escritura colaborativo, en la que el tutor participa en la resolución de las tareas de escritura, monitorea el proceso del estudiante y apoya su toma de decisiones fundamentadas. En efecto, en las sesiones de tutorías, los tutores suelen destacar la relevancia de fomentar un buen vínculo con los estudiantes y el de estos últimos con sus escritos, el trabajo cooperativo entre ambas partes y la comunicación adecuada de sus reflexiones a los tutorados (Harrington et al., 2007).

En este sentido, de acuerdo con Jones et al. (2006), las tutorías en línea de escritura académica en inglés como segunda lengua tienden a ser menos jerárquicas, más igualitarias y menos directivas que las presenciales. Lo anterior es confirmado por Hewett (2006) en el ámbito anglosajón, dado que los cambios positivos en la escritura y la revisión de los textos radicaron en la interacción en línea colaborativa y menos instructiva entre tutor y estudiante. Sin embargo, en el contexto hispánico, las tutorías presenciales han sido más valoradas, dada la posibilidad de establecer una relación directa y personal más valiosa para el desarrollo académico, según el alumnado (Martínez et al., 2016). Por el contrario, Holtz (2014) plantea que las tutorías en línea son una alternativa viable que ofrece apoyos y recursos que pueden ser incluso más eficaces. En consecuencia, cabría indagar qué ocurre en el ámbito hispanohablante al respecto, especialmente si consideramos que las distintas experiencias en relación con tutorías virtuales, sobre todo en la esfera angloparlante, dan cuenta de que son instancias necesarias y deseables para los estudiantes como parte de los servicios que las universidades debieran brindarles para fortalecer el proceso de escritura (Núñez y García, 2018).

A propósito del punto anterior sobre la comunicación con los estudiantes, la investigación sobre la retroalimentación de las tareas de escritura se ha enfocado principalmente en el tipo de comentarios que hacen los profesores en sus devoluciones escritas (Natale, 2016) o los propios alumnos en actividades de revisión de pares en el contexto de los cursos de las carreras. Algunas evidencias exponen el modo en que la retroalimentación que pone el foco en recomendaciones y preguntas provoca una transformación más significativa o profunda 
que aquella generada por comentarios directivos (Álvarez et al., 2011).

\section{Estrategias didácticas de las tutorías de escritura}

En relación con las estrategias didácticas para llevar a cabo las tutorías, estas han sido sistematizadas y caracterizadas en varios estudios (Caldwell et al., 2018; Cromley y Azevedo, 2005; Eastmond, 2019; Mackiewicz, 2004, 2005; Mackiewicz y Thompson, 2014, 2018; Roldán y Arenas, 2016). A partir de estas propuestas, nos parece particularmente relevante la planteada por Mackiewicz y Thompson $(2014,2018)$, debido a su precisión en la categorización. Las autoras plantean dos niveles de análisis: en primer lugar, el macronivel, en donde existen las fases de apertura, desarrollo y cierre; y, en segundo lugar, el micronivel, que presenta tres dimensiones: la instrucción, el andamiaje cognitivo y el andamiaje motivacional. Con respecto a sus resultados, ellas identificaron un predominio de estrategias instruccionales en diez tutorías analizadas llevadas a cabo por tutores experimentados (Mackiewicz y Thompson, 2014, 2018).

En el contexto del micronivel, en el que nos centramos en este estudio, la instrucción es principalmente directiva, debido a que en ella se señala, recomienda o explica qué hacer, y no se promueven oportunidades para que los estudiantes resuelvan los problemas por sí mismos.

En oposición a la estrategia anterior, la de andamiaje cognitivo - propia de tutores más experimentados - fomenta la exploración de soluciones mediante la activación del pensamiento, la reflexión y el diálogo entre el tutor y el estudiante, es decir, a través de la mediación cognitiva del tutor, que puede manifestarse por medio de ocho estrategias: jalonar, leer en voz alta, responder como lector u oyente, mencionar un tema anterior, forzar una elección, incitar, insinuar o sugerir, y demostrar (Molina-Natera, 2019). En efecto, según Calle-Arango (2019), la formulación de preguntas complejas en la instancia de tutoría promueve el aprendizaje profundo y crítico de los estudiantes sobre sus procesos de escritura.

Por último, las estrategias de andamiaje motivacional consideran las dimensiones emocionales y afectivas de la tutoría y del proceso de escritura, por lo que los tutores muestran afecto hacia el estudiante o sus logros, para así estimular su participación y confianza, elevar su autoconcepto como escritor y construir un vínculo positivo con el tutorado. Estas estrategias se manifiestan al mostrar interés, elogiar, reforzar la posesión y el autocontrol del tutorado, ser optimista o mostrar humor, y expresar simpatía y empatía (véase Figura 1).
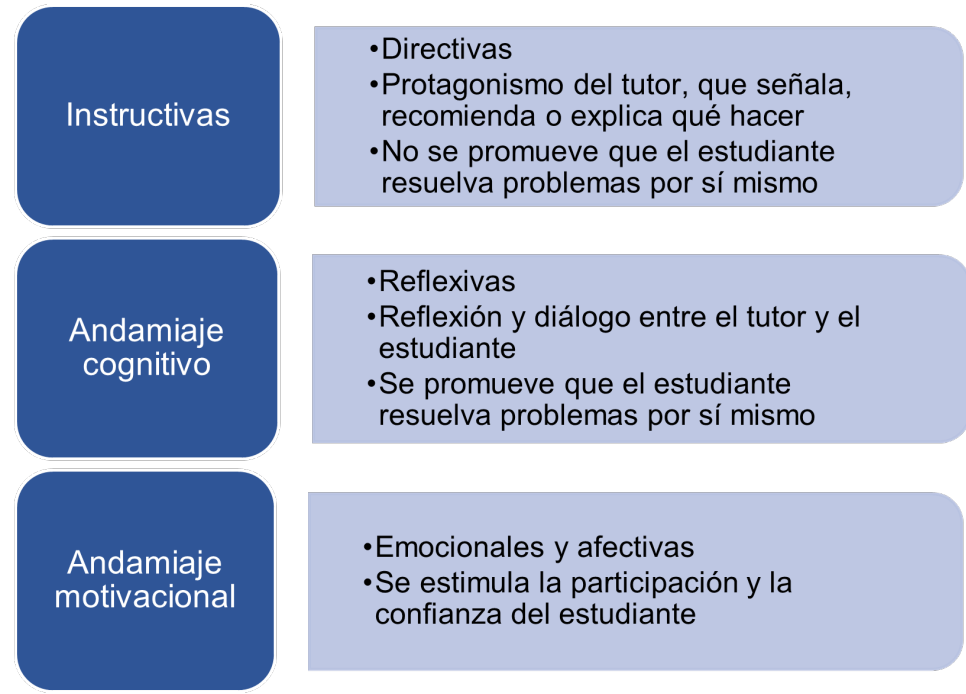

Figura 1 Características de los tipos de estrategias en las tutorías de escritura 


\section{Método}

Esta investigación se encuadra dentro de un paradigma de corte mixto, cuantitativo- cualitativo, que se efectuó mediante un análisis de contenido. Esta metodología dispone de tres fases principales (Assarroudi et al., 2018): preparación, organización y presentación. En la primera, se selecciona la muestra y se desarrollan las tutorías, que son grabadas y posteriormente transcritas. En la segunda se establece la matriz de análisis y se definen teóricamente las categorías y los códigos respecto de las estrategias didácticas de las tutorías, que se describirán más adelante. También en esta fase se realiza el análisis de los textos transcritos, que previamente se sometieron a una prueba piloto de codificación. Por último, en la fase de presentación, se exponen los hallazgos del estudio.

Este enfoque ha sido aplicado anteriormente por otras investigaciones que han analizado las tutorías de escritura en centros de escritura (Cromley y Azevedo, 2005; Eastmond, 2019; Mackiewicz, 2004, 2005; Mackiewicz y Thompson, 2014, 2018; Roldán y Arenas, 2016), por considerarse adecuado para comprender la dinámica propia de las tutorías de escritura y la interacción entre tutor y estudiante.

\section{Participantes}

En las tutorías participaron 16 estudiantes y 2 tutoras, que fueron informados del objetivo de la investigación, y todos expresaron su consentimiento explícito, escrito en el caso de las tutoras y oral, registrado mediante grabación, en el caso de los estudiantes.

Las tutorías se realizaron en el Centro de Escritura de la Facultad de Formación de Profesorado y Educación de la Universidad Autónoma de Madrid.

Los estudiantes de las tutorías presenciales cursaban 2. ${ }^{\circ}$ año del Grado Maestro/a de Educación Primaria; los de las virtuales, $3 .^{\text {er }}$ año del Doble Grado de Maestro/a de Educación Infantil y Educación Primaria.
En cuanto a las tutoras, la tutora experta (TE) cursaba el último año $\left(4 .^{\circ}\right)$ del Grado de Maestro/a de Educación Primaria en el curso 2019/2020, y en el momento de la recogida de datos había impartido 100 tutorías. Por otra parte, la tutora novel (TN) cursaba 4. ${ }^{\circ}$ año del Grado de Maestro/a de Educación Infantil y había impartido 27 tutorías.

Las dos tutoras habían recibido previamente una formación de 40 horas de duración en el Centro de Escritura para desempeñarse como tales, centrada en tres módulos: 1) el centro de escritura; 2) el proceso de escritura y los géneros discursivos académicos, y 3) las tutorías.

\section{Recolección y análisis de los datos}

En la investigación, se han analizado las transcripciones de 16 tutorías individuales de media hora de duración, 8 presenciales grabadas en audio y 8 virtuales realizadas a través de videollamada mediante Microsoft Teams. Las tutorías presenciales se hicieron durante el primer cuatrimestre (septiembre a enero) del curso 2019/2020; las virtuales, entre el segundo cuatrimestre (febrero a mayo) del curso 2019/2020 y el primer cuatrimestre del curso 2020/2021.

El cambio de modalidad de tutoría presencial a virtual en el Centro de Escritura se produjo en el mes de marzo de 2020, fecha en la que se decretó el estado de alarma que imponía el confinamiento domiciliario por la COVID-19 y, en consecuencia, las universidades españolas adaptaron su modelo de enseñanza al entorno virtual.

Fueron dos los criterios de selección de los textos de las tutorías: por un lado, eran borradores de la versión final, por lo que se trabajó la fase de revisión del proceso de escritura; por otro, eran ensayos argumentativos, pues este género discursivo es frecuente en los programas de formación docente (Castro et al., 2010; Errázuriz, 2019; Parodi, 2008), lo que es relevante en este contexto, dado que gran parte del desafío cognitivo que implica la literacidad académica se funda en la capacidad de argumentar (Muñoz y Valenzuela, 2015; Van Eemeren, 2001). 
Además, la reflexión es una habilidad crucial para la práctica docente (Sun et al., 2011).

Para analizar las estrategias didácticas de las tutorías, se estableció una serie de categorías (véase Tabla 1) a partir de los trabajos de Mackiewicz y Thompson $(2014,2018)$ y de otras investigaciones (Caldwell et al., 2018; Cromley y Azevedo, 2005; Eastmond, 2019; Mackiewicz, 2005; Roldán y Arenas, 2016; Thompson, 2009). Posteriormente, se efectuó un análisis inductivo-deductivo, mediante la lectura analítica de los textos transcritos. También se establecieron tres tipos de estrategias (instructivas, cognitivas y motivacionales), que concuerdan en términos generales con la propuesta de Mackiewicz y Thompson (2018): instrucción, andamiaje cognitivo y andamiaje motivacional. Cabe señalar que, a partir del trabajo de Mackiewicz y Thompson (2018), algunas de sus estrategias se han matizado o atenuado, y otras son nuevas; además, presentamos ejemplos propios.

Tabla 1 Estrategias didácticas de las tutorías de escritura (matriz de análisis)

\begin{tabular}{|c|c|c|c|}
\hline \multicolumn{2}{|c|}{ Tipos de estrategias } & Descripción & Ejemplo \\
\hline \multirow{3}{*}{$\begin{array}{l}\text { Estrategias } \\
\text { instructivas }\end{array}$} & Explicación & $\begin{array}{l}\text { Se ofrecen razones, ilustraciones o ejemplos } \\
\text { de los consejos ofrecidos }\end{array}$ & $\begin{array}{l}\text { Tutora: "Todo lo que has quitado lo puedes } \\
\text { resumir, o sea, puedes decir la misma idea } \\
\text { con menos palabras" }\end{array}$ \\
\hline & Orientación & $\begin{array}{l}\text { Se indica o aconseja al estudiante sobre lo que } \\
\text { ha de hacer de forma directa, no mitigada }\end{array}$ & Tutora: "Estás repitiendo esta idea" \\
\hline & Sugerencia & $\begin{array}{l}\text { Se indica o aconseja al estudiante sobre lo que ha } \\
\text { de hacer de forma atenuada, minimizando así la } \\
\text { indicación (p. ej. a través de la cortesía negativa) }\end{array}$ & Tutora: "Yo la quitaría" \\
\hline \multirow{8}{*}{$\begin{array}{l}\text { Estrategias } \\
\text { cognitivas }\end{array}$} & Completamiento & $\begin{array}{l}\text { Se incita a que el estudiante responda, } \\
\text { proporcionándole una respuesta o enunciado } \\
\text { parcial, y así reducir las posibles respuestas } \\
\text { (p. ej. rellenar un espacio en blanco) }\end{array}$ & $\begin{array}{l}\text { Tutora: "Trasmitirle una idea... } \\
\text { planteada...". Estudiante: "En una reunión" }\end{array}$ \\
\hline & Comprobación & $\begin{array}{l}\text { Se comprueba la comprensión del estudiante } \\
\text { de algo ya tratado, mediante una interrogación } \\
\text { o la solicitud de una explicación }\end{array}$ & Tutora: “iEntiendes?”, “iMe explico?” \\
\hline & Elección & $\begin{array}{l}\text { Se ofrecen varias alternativas para que se } \\
\text { escoja una }\end{array}$ & $\begin{array}{l}\text { Estudiante: "No tiene uso, más bien". } \\
\text { Tutora: "Está en desuso", "no se utiliza" }\end{array}$ \\
\hline & Finalidad & $\begin{array}{l}\text { Se establece o se busca establecer el objetivo } \\
\text { de la tutoría. Se recuerda el objetivo de la } \\
\text { tutoría }\end{array}$ & $\begin{array}{l}\text { Tutora: "Vale, entonces, no te interesa quizá } \\
\text { tanto la redacción del texto en sí, como saber } \\
\text { reducir la extensión" }\end{array}$ \\
\hline & Interpretación & $\begin{array}{l}\text { Se interpreta el texto del estudiante, es decir, } \\
\text { se muestra qué se entiende del fragmento } \\
\text { referido. Se parafrasea el texto }\end{array}$ & $\begin{array}{l}\text { Tutora: "Yo, como lectora, al leer esto, me } \\
\text { planteo si existe otro gimnasio que no sea el } \\
\text { de la planta de abajo" }\end{array}$ \\
\hline & $\begin{array}{l}\text { Lectura del } \\
\text { estudiante en voz } \\
\text { alta }\end{array}$ & $\begin{array}{l}\text { El estudiante lee en voz alta fragmentos de su } \\
\text { texto con el objetivo de identificar errores de } \\
\text { contenido o forma }\end{array}$ & $\begin{array}{l}\text { Tutora: "Pues si te parece, lo vas leyendo tú, } \\
\text { que así también te puedes ir dando cuenta } \\
\text { mejor de los errores". (Estudiante lee su texto) }\end{array}$ \\
\hline & $\begin{array}{l}\text { Lectura del tutor } \\
\text { en voz alta }\end{array}$ & $\begin{array}{l}\text { Se leen, en voz alta, fragmentos del texto } \\
\text { del estudiante, para escuchar lo escrito o la } \\
\text { consigna de la tarea de escritura, con el fin de } \\
\text { verificar la comprensión del estudiante }\end{array}$ & $\begin{array}{l}\text { Tutora: "Te leo: 'es para transmitirle una idea } \\
\text { planteada en un claustro. La idea que hemos } \\
\text { planteado [...]". }\end{array}$ \\
\hline & Marcación & $\begin{array}{l}\text { Se señala explícitamente un problema, error } 0 \\
\text { aspecto del texto que ha de atenderse }\end{array}$ & $\begin{array}{l}\text { Tutora: "En esa primera oración que has escrito } \\
\text { hay algo que falta relacionado con la puntuación" }\end{array}$ \\
\hline
\end{tabular}


Tabla 1 Estrategias didácticas de las tutorías de escritura (matriz de análisis) (cont.)

\begin{tabular}{|c|c|c|c|}
\hline \multicolumn{2}{|c|}{ Tipos de estrategias } & \multirow{2}{*}{$\begin{array}{l}\text { Descripción } \\
\text { Se muestra al estudiante un texto modelo, } \\
\text { una guía de escritura o una rúbrica, para que } \\
\text { aprenda a hacer algo }\end{array}$} & \multirow{2}{*}{$\begin{array}{l}\text { Ejemplo } \\
\text { Tutora: "Mira cómo se plantea en este } \\
\text { ejemplo, que es prácticamente igual, pues es } \\
\text { un texto argumentativo". }\end{array}$} \\
\hline \multirow{7}{*}{$\begin{array}{l}\text { Estrategias } \\
\text { cognitivas }\end{array}$} & Soporte & & \\
\hline & Pregunta & $\begin{array}{l}\text { Se formulan una o varias preguntas que } \\
\text { requieren de la respuesta del estudiante. Las } \\
\text { preguntas pueden ser específicas o generales }\end{array}$ & Tutora: ¿Por qué cambias de párrafo?” \\
\hline & Recomendación & $\begin{array}{l}\text { Se recomienda al estudiante el uso de un } \\
\text { recurso para que resuelva el problema, error } 0 \\
\text { aspecto tratado }\end{array}$ & $\begin{array}{l}\text { Tutora: "En la web del centro de escritura hay } \\
\text { un recurso sobre cómo escribir un estado de } \\
\text { la cuestión" }\end{array}$ \\
\hline & Reescritura & $\begin{array}{l}\text { Se plantea al estudiante que reescriba un } \\
\text { fragmento que contiene un problema, error } 0 \\
\text { aspecto que debe ser atendido }\end{array}$ & $\begin{array}{l}\text { Tutora: "Pues entonces, si no te convence, } \\
\text { reescríbelo como me acabas de decir, y luego } \\
\text { vemos cómo queda" }\end{array}$ \\
\hline & Remisión & $\begin{array}{l}\text { Se remite a un tema, error, problema } 0 \\
\text { aspecto del texto ya tratado }\end{array}$ & $\begin{array}{l}\text { Tutora: "Pero eso ya lo hemos mencionado } \\
\text { anteriormente" }\end{array}$ \\
\hline & Reticencia & $\begin{array}{l}\text { Se hace referencia o se plantea un problema, } \\
\text { error } 0 \text { aspecto del texto, mediante una } \\
\text { estrategia de cortesía indirecta no convencional }\end{array}$ & $\begin{array}{l}\text { Tutora: "Aun así, lo que yo te quería decir } \\
\text { es que si tú dices: 'porque, según como } \\
\text { argumentan...', así no suena muy bien" }\end{array}$ \\
\hline & Solución & $\begin{array}{l}\text { Se ofrece la solución al problema, error } 0 \\
\text { aspecto del texto }\end{array}$ & $\begin{array}{l}\text { La tutora lee el texto e indica el cambio: "El } \\
\text { motivo por el que nos ponemos en contacto } \\
\text { con usted es para transmitirle una idea [coma], } \\
\text { que hemos debatido en el claustro [...]" }\end{array}$ \\
\hline \multirow{7}{*}{$\begin{array}{l}\text { Estrategias } \\
\text { motivadoras }\end{array}$} & Ánimo/humor & $\begin{array}{l}\text { Se anima al estudiante a perseverar en la } \\
\text { tarea y mejorar el texto. Se utiliza el humor, } \\
\text { mediante bromas relacionadas con el texto o } \\
\text { el contexto de la tutoría }\end{array}$ & $\begin{array}{l}\text { Tutora: "Esa tiene tela [que cortar]" (Tras leer } \\
\text { una oración, ininteligible, tutor y estudiante } \\
\text { se ríen antes y después) }\end{array}$ \\
\hline & Elogio & $\begin{array}{l}\text { Se señalan los aspectos positivos del texto } 0 \\
\text { de la resolución de los problemas por parte } \\
\text { del estudiante }\end{array}$ & Tutora: "Mucho mejor así. Perfecto" \\
\hline & Empatía & $\begin{array}{l}\text { Se expresa la compresión de que la tarea es } \\
\text { difícil }\end{array}$ & $\begin{array}{l}\text { Tutora: "Este tipo de texto siempre es } \\
\text { complicado" }\end{array}$ \\
\hline & Identificación & $\begin{array}{l}\text { Se hace referencia a experiencias propias } \\
\text { relacionadas con la escritura de textos } \\
\text { similares }\end{array}$ & $\begin{array}{l}\text { Tutora: "Eso es una duda que yo también } \\
\text { suelo tener a veces". } \\
\text { Tutora: "Claro. Yo lo hago igual" }\end{array}$ \\
\hline & Interés & $\begin{array}{l}\text { Se muestra el interés por el estado emocional } \\
\text { del estudiante }\end{array}$ & $\begin{array}{l}\text { Tutora: "¿Ves que no es tan difícil? ¿Estás } \\
\text { mejor?" }\end{array}$ \\
\hline & Refuerzo & $\begin{array}{l}\text { Se incide en que es el estudiante quien debe } \\
\text { tomar las decisiones }\end{array}$ & $\begin{array}{l}\text { Tutora: "Entonces, ahora ahí, haces las } \\
\text { modificaciones que consideres". } \\
\text { Tutora: "Lo dejo a tu elección" }\end{array}$ \\
\hline & Significación & $\begin{array}{l}\text { Se mencionan los aspectos aprendidos } 0 \\
\text { trabajados durante o al final de la tutoría, } \\
\text { recapitulando lo tratado. Se hace referencia a } \\
\text { la relevancia que puede tener lo aprendido en } \\
\text { la escritura de futuros textos }\end{array}$ & $\begin{array}{l}\text { Tutora: "Así no estaba en tu texto; esto de la } \\
\text { coma entre sujeto y verbo no lo ponías así, y } \\
\text { ahora sí". }\end{array}$ \\
\hline
\end{tabular}

Fuente: elaboración propia, con base en Mackiewicz y Thompson (2018). 
Tabla 2 Frecuencia y porcentaje de estrategias utilizadas en las tutorías

\begin{tabular}{lcccccc}
\hline \multirow{2}{*}{ Estrategias } & \multicolumn{2}{c}{ Tutorías presenciales } & \multicolumn{2}{c}{ Tutorías virtuales } & \multicolumn{2}{c}{ Total } \\
\cline { 2 - 7 } & $\mathbf{F i}$ & $\%$ & $\mathbf{F i}$ & $\%$ & $\mathbf{F i}$ & $\%$ \\
\hline Instructivas & 363 & 35,21 & 305 & 30,41 & 668 & 32,84 \\
Cognitivas & 564 & 54,70 & 530 & 52,84 & 1094 & 53,79 \\
Motivadoras & 104 & 10,09 & 168 & 16,75 & 272 & 13,37 \\
Total & 1031 & 100 & 1003 & 100 & 2034 & 100 \\
\hline
\end{tabular}

Fi: Frecuencia.

Cada tutoría fue analizada en dos ocasiones por dos investigadores diferentes. Esto permitió triangular y calibrar la codificación, o lo que es lo mismo, señalar, en el software de análisis mixto MAXQDA Analytics Pro 2020, el uso de las estrategias empleadas por las tutoras y así conocer la frecuencia y la relación entre cada una de ellas.

En cuanto a los análisis, la primera técnica que se realizó fue una matriz de códigos por conjunto de documentos, diferenciando estos según el tipo de tutoría (presencial versus virtual), la tutora (experta versus novel) y los datos totales. Así, se pudo conocer con qué frecuencia se emplearon las estrategias en cada uno de los casos mencionados anteriormente.

Por otro lado, el segundo análisis consistió en un modelo de dos casos entre las tutorías presenciales y virtuales, con el fin de discernir las cinco estrategias más utilizadas en cada tipo de tutoría.

\section{Resultados}

A continuación se presentan los resultados del estudio. En primer lugar, se da cuenta de las estrategias utilizadas en las tutorías presenciales y virtuales. Posteriormente, se muestran los resultados en función del tipo de estrategias (instructiva, cognitiva y motivacional). Por último, se atiende a cómo se relacionan las estrategias más utilizadas en las tutorías.

A partir del análisis efectuado, y con relación al objetivo principal de conocer cuáles son las estrategias didácticas más utilizadas en las tutorías presenciales y virtuales, en total predominan las estrategias cognitivas, con el 53,79\% de intervenciones, seguidas de las instructivas (32, $84 \%$ ) y las motivadoras (13,37 \%) (véase Tabla 2). Esto muestra que existe una clara consciencia por parte de las tutoras de primar el andamiaje cognitivo, pues estas estrategias constituyen más de la mitad de las empleadas tanto en las tutorías presenciales como en las virtuales. No obstante, se percibe cómo estos resultados varían en función del tipo de tutoría. Así, se produce un descenso en las estrategias instructivas $(4,8 \%)$ y cognitivas $(1,86 \%)$ en las tutorías virtuales en favor de las estrategias motivadoras $(6,66 \%)$.

En cuanto a las estrategias instructivas, la más empleada en el total de los dos tipos de tutoría es la sugerencia $(43,56 \%)$, seguida de la explicación $(32,33 \%)$ y la orientación (24,10 \%). De hecho, esta primera estrategia es la única que aumenta en las tutorías virtuales respecto de las presenciales, ya que de 126 asciende a 165 códigos (véase Tabla 3). Frente a esto, tanto la explicación (-42,33\%) como la orientación $(-39 \%)$ disminuyen en las sesiones virtuales.

Con relación a las tutoras, la TN emplea la sugerencia casi el doble de veces que la TE en las tutorías virtuales, diferencia que si bien existía en las presenciales, no era tan acusada. Por otro lado, esta situación se revierte en el uso de la orientación y la explicación, puesto que si bien las dos tutoras reducen la cantidad de códigos en las instancias en línea, esta disminución es mayor en la TN.

Las estrategias cognitivas son, como se ha indicado, las más utilizadas y, tras el análisis, se pueden 
Tabla 3 Frecuencia de estrategias según tutor y tipo de tutoría (matriz de códigos por conjunto de documentos)

\begin{tabular}{|c|c|c|c|c|c|c|c|c|c|c|}
\hline & \multirow[t]{2}{*}{ Estrategias } & \multicolumn{3}{|c|}{ Tutorías presenciales } & \multicolumn{3}{|c|}{ Tutorías virtuales } & \multicolumn{3}{|c|}{ Total } \\
\hline & & TE & TN & Tt & TE & TN & Tt & $\mathrm{TE}$ & TN & Tt \\
\hline & Explicación & 62 & 75 & 137 & 39 & 40 & 79 & 101 & 115 & 216 \\
\hline \multirow[t]{2}{*}{ Instructivas } & Orientación & 36 & 64 & 100 & 24 & 37 & 61 & 60 & 101 & 161 \\
\hline & Sugerencia & 53 & 73 & 126 & 57 & 108 & 165 & 110 & 181 & 291 \\
\hline \multirow{15}{*}{ Cognitivas } & Completamiento & 10 & 5 & 15 & 4 & 8 & 12 & 14 & 13 & 27 \\
\hline & Comprobación & 15 & 21 & 36 & 16 & 2 & 18 & 31 & 23 & 54 \\
\hline & Elección & 12 & 30 & 42 & 12 & 23 & 35 & 24 & 53 & 77 \\
\hline & Finalidad & 5 & 1 & 6 & 6 & 3 & 9 & 11 & 4 & 15 \\
\hline & Interpretación & 29 & 21 & 50 & 19 & 14 & 33 & 48 & 35 & 83 \\
\hline & Lectura del estudiante & 47 & 3 & 50 & 28 & 5 & 33 & 75 & 8 & 83 \\
\hline & Lectura de la tutora & 24 & 66 & 90 & 23 & 45 & 68 & 47 & 111 & 158 \\
\hline & Marcación & 17 & 23 & 40 & 26 & 44 & 70 & 43 & 67 & 110 \\
\hline & Pregunta & 48 & 59 & 107 & 69 & 68 & 137 & 117 & 127 & 244 \\
\hline & Recomendación & 9 & 4 & 13 & 13 & 23 & 36 & 22 & 27 & 49 \\
\hline & Reescritura & 0 & 3 & 3 & 9 & 1 & 10 & 9 & 4 & 13 \\
\hline & Remisión & 6 & 1 & 7 & 6 & 9 & 15 & 12 & 10 & 22 \\
\hline & Reticencia & 14 & 20 & 34 & 8 & 12 & 20 & 22 & 32 & 54 \\
\hline & Solución & 9 & 45 & 54 & 18 & 16 & 34 & 27 & 61 & 88 \\
\hline & Soporte & 17 & 0 & 17 & 0 & 0 & 0 & 17 & 0 & 17 \\
\hline \multirow{7}{*}{ Motivacionales } & Ánimo/Humor & 3 & 4 & 7 & 12 & 9 & 21 & 15 & 13 & 28 \\
\hline & Elogio & 25 & 43 & 68 & 23 & 40 & 63 & 48 & 83 & 131 \\
\hline & Empatía & 1 & 0 & 1 & 7 & 4 & 11 & 8 & 4 & 12 \\
\hline & Identificación & 4 & 10 & 14 & 16 & 6 & 22 & 20 & 16 & 36 \\
\hline & Interés & 0 & 0 & 0 & 10 & 1 & 11 & 10 & 1 & 11 \\
\hline & Refuerzo & 2 & 6 & 8 & 12 & 13 & 25 & 14 & 19 & 33 \\
\hline & Significación & 2 & 4 & 6 & 6 & 9 & 15 & 8 & 13 & 21 \\
\hline Total & & 450 & 581 & 1031 & 463 & 540 & 1003 & 913 & 1121 & 2034 \\
\hline
\end{tabular}

TE: Tutora experta; TN: tutora novel; Tt: suma de ambas.

clasificar en tres grupos, en función de su uso: 1) abundante (>10\% respecto del total de las estrategias cognitivas), 2) moderado $(5<x<$ $9,99 \%)$ y 3$)$ escaso $(0<x<4,99 \%)$. En el primer grupo se encuentran las estrategias de la pregunta $(22,33 \%)$, la lectura del tutor $(14,44 \%)$ y la marcación (10,05\%). Asimismo, es moderado el uso de la elección (7,03\%), la interpretación (7,58 \%), la lectura del estudiante $(7,58 \%)$ y la solución $(8,04 \%)$. Por último, el uso del resto de estrategias se enmarcaría en el tercer grupo; así, son escasas, entre otras, las estrategias de reescritura, finalidad y soporte.

Por otro lado, si bien existía una leve disminución de las estrategias cognitivas en las tutorías virtuales, se percibe un considerable aumento de algunas de las estrategias de esta categoría, como: finalidad, marcación, pregunta, recomendación, reescritura y remisión. Al respecto, este incremento es especialmente notorio en la marcación, la pregunta y la recomendación, tanto en la TN como en la experta. 
Asimismo, el resto de estrategias disminuye en las tutorías virtuales y este descenso se percibe relevante en las estrategias de lectura del tutor, solución y soporte. Estos datos coinciden con los resultados en función de las tutoras, salvo en el caso de la solución, puesto que la TE aumenta el número de códigos de esta estrategia en las tutorías virtuales. El descenso es notorio, pues dado el considerable menor uso de la solución por parte de la TN en las tutorías virtuales, su frecuencia es del $-64,44 \%$.

En relación con las estrategias motivadoras, la más utilizada es el elogio $(48,16 \%)$, con mucha diferencia respecto de las demás, cuyo uso es moderadamente equilibrado, a excepción de las estrategias de empatía e interés. Como se indicó anteriormente, las estrategias motivadoras son las únicas que aumentan en las tutorías virtuales.

Llama la atención al respecto cómo — salvo la identificación - todas las estrategias aumentan considerablemente, de tal manera que en las tuto- la identificación, el interés, el refuerzo y la significación. Curiosamente, el elogio — pese a ser la más utilizada - es la única estrategia que disminuye de las siete estrategias de esta categoría. Por otra parte, en cuanto a la diferencia entre la TN y la $\mathrm{TE}$, los resultados muestran que la primera emplea el elogio casi el doble de veces que la segunda. Sin embargo, la TE muestra más empatía e interés por los estudiantes.

Dado que las estrategias más utilizadas varían en función del tipo de tutoría, resulta interesante saber cómo se relacionan estas con el resto de estrategias más empleadas en las tutorías presenciales y virtuales. Como se aprecia en la Figura 2 , en las tutorías presenciales, las estrategias más utilizadas en función de su categoría son: la explicación (instructivas), la pregunta (cognitivas) y el elogio (motivadoras). Sin embargo, en las tutorías virtuales - si bien las estrategias cognitivas y motivadoras son las mismas - predomina la estrategia instructiva de la sugerencia frente a la explicación. Estos datos contemplan diferentes

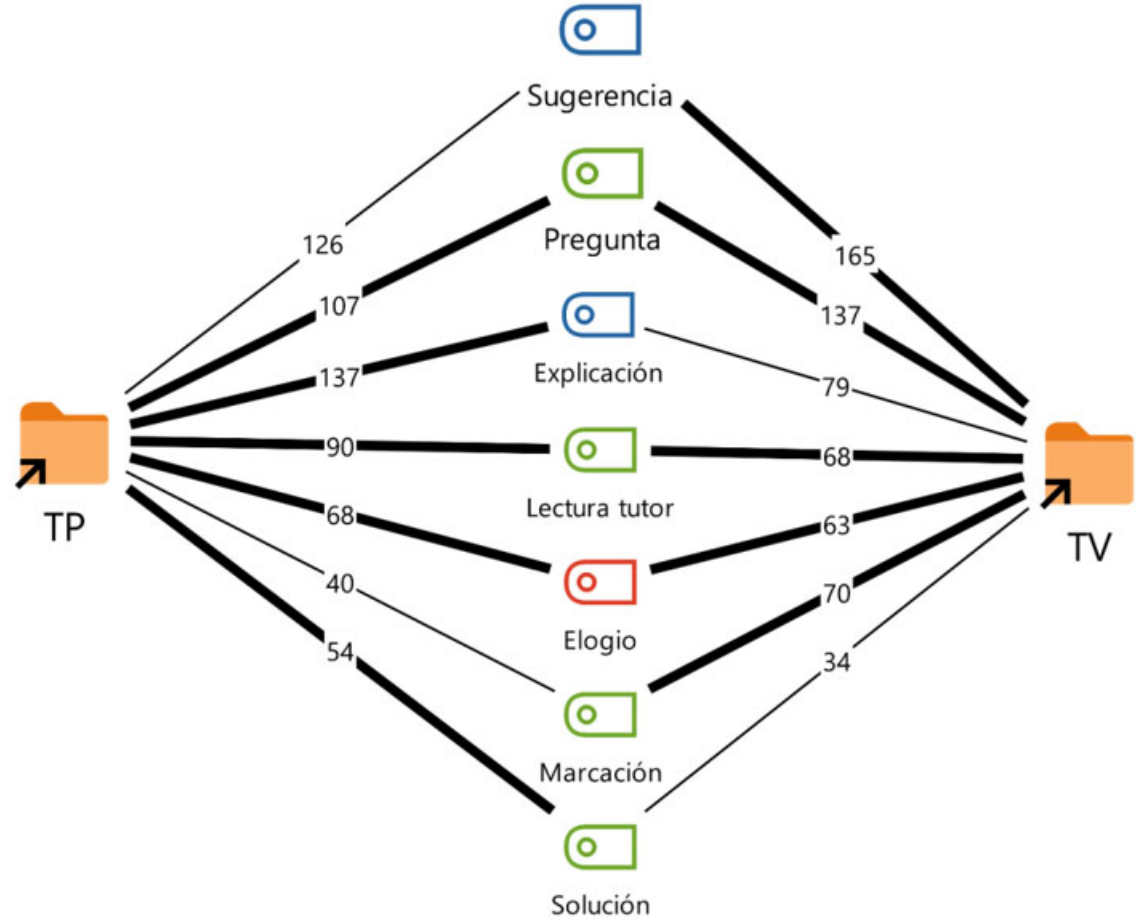

Figura 2 Estrategias más utilizadas en tutorías presenciales (TP) y virtuales (TV) (modelo de casos). Convenciones: azul: instructivas; verde: cognitivas; naranja: motivadoras. 
matices que no se pueden dejar de atender. Así, es pertinente señalar que la orientación y la lectura del tutor son estrategias muy utilizadas en ambos tipos de tutorías. También llama la atención que en las tutorías presenciales predomine la solución, respecto a la marcación en las tutorías virtuales.

A partir de los datos, se puede afirmar que las estrategias más utilizadas permiten observar un modelo de tutoría en donde predomina la sugerencia o la explicación (y en ocasiones la orientación), la pregunta, la lectura de la tutora y, por último, la marcación o la solución. A modo de ejemplo, en la Tabla 4 se muestra una secuencia prototípica de una tutoría por parte de la TE.

\section{Discusión y conclusiones}

El objetivo de las tutorías de escritura es desarrollar la competencia en expresión escrita de los estudiantes universitarios a partir de sus propios textos. Para ello, se promueve la reflexión, como consecuencia de la puesta en práctica de estrategias didácticas por parte de un tutor.

$\mathrm{Al}$ respecto, estas estrategias se podrían asociar con dos modelos dicotómicos de tutorías, uno centrado en el estudiante y el proceso de escritura, y otro centrado en el tutor y el producto. No obstante, algunos factores como la propia competencia escrita del estudiante, la calidad del texto trabajado o el nivel de experticia del tutor sugieren abogar por un modelo de tutoría híbrida, que permita no asociar unas estrategias a un modelo concreto y "correcto" de tutoría.

En este sentido, el grado de frecuencia de aparición de algunas estrategias no tiene por qué ser elevado. Por ejemplo, la estrategia de finalidad no es necesario que se utilice continuamente; bastaría con contemplarla al inicio de la tutoría o recuperarla en el caso de que el objetivo se difuminara durante la sesión. Por el contrario, llamaría la atención que en un modelo de tutoría que propicie la reflexión y el diálogo no hubiera una presencia considerable de estrategias como la pregunta, la interpretación, la explicación o la sugerencia.

En cuanto a los resultados del trabajo presentado, como se ha indicado, existe una tendencia tanto en las tutorías presenciales como en las virtuales a utilizar en mayor grado las estrategias cognitivas, seguidas de las instructivas y motivadoras. En este sentido, por un lado, los resultados son

Tabla 4 Secuencia prototípica de estrategias de una tutora experta

\begin{tabular}{|c|c|}
\hline Fragmento de tutoría & Estrategia y explicación \\
\hline $\begin{array}{l}\text { Estudiante: "Pero no explicamos esa idea como tal, sino que la } \\
\text { hemos explicado en el siguiente párrafo y yo creo que habría que } \\
\text { introducirla aquí" }\end{array}$ & $\begin{array}{l}\text { El estudiante reflexiona sobre dónde y cómo plantear una idea en } \\
\text { el texto }\end{array}$ \\
\hline $\begin{array}{l}\text { Tutora: "Mmmm... Vale. A ver, yo creo que lo que podríais hacer, } \\
\text { para empezar, antes de ver cómo juntar esos dos párrafos, es decir, } \\
\text { la misma información, pero con menos 'eee' redacción. }\end{array}$ & $\begin{array}{l}\text { La tutora emplea la estrategia de la sugerencia. Plantea al } \\
\text { estudiante la posibilidad de transmitir la idea de forma más breve, } \\
\text { con menos palabras }\end{array}$ \\
\hline $\begin{array}{l}\text { Tutora: "Tú dices: 'El motivo por el que nos ponemos en contacto con } \\
\text { usted, [...]', haces una pausa, 'es para transmitirle una idea..." }\end{array}$ & $\begin{array}{l}\text { La tutora emplea la estrategia de lectura en voz alta por parte ella } \\
\text { y la marcación para señalar un error de puntuación: el uso de la } \\
\text { coma entre sujeto y verbo }\end{array}$ \\
\hline Tutora: “¿Crees que está bien puntuada esa oración?" & $\begin{array}{l}\text { La tutora plantea una pregunta como estrategia para cuestionar al } \\
\text { estudiante si el uso de la coma es correcto }\end{array}$ \\
\hline Estudiante: "No. Hay que quitar la coma, no hace falta la pausa" & La estudiante responde a la pregunta \\
\hline Tutora: "0 sea, nunca puede separar una coma sujeto de predicado" & $\begin{array}{l}\text { La tutoría refuerza la respuesta de la estudiante mediante la } \\
\text { estrategia de explicación }\end{array}$ \\
\hline
\end{tabular}


similares a los de investigaciones precedentes (Mackiewicz y Thompson, 2014, 2018), dado que las estrategias más frecuentes son la explicación, la sugerencia y la pregunta. No obstante, por otro, en sus investigaciones, estas autoras muestran cómo predominan las estrategias instructivas seguidas de las cognitivas.

Es necesario, por tanto, indagar en las causas y explicaciones de las diferencias de estos resultados, pues han sido recabados en distintos contextos. Una de ellas se puede deber a la formación de los tutores y su ámbito de estudio, ya que las tutoras y tutorados de la presente investigación son estudiantes de programas de formación docente y el centro de escritura es parte de una Facultad de Formación de Profesorado y Educación, en contraste con los tutores que son estudiantes de lengua inglesa de diferentes carreras en el caso de la investigación de Mackiewicz y Thompson. Por tanto, la formación de las tutoras y las tutorías analizadas en el presente estudio pueden tener un mayor foco en la construcción colaborativa y dialógica de aprendizajes, en el andamiaje y en la motivación. Sin embargo, es necesario comprobar con mayores evidencias estas posibles causas.

Con relación a las estrategias cognitivas, conviene subrayar que tanto la estrategia de pregunta como la de lectura del tutor predominan en ambas tutorías, con lo que se busca impulsar al tutorado a percibir los aciertos y falencias de su texto. De hecho, la formulación de preguntas por parte del tutor es una estrategia útil para la promoción de la reflexión y la colaboración (Calle-Arango, 2019). Así, se puede deducir que juntos, tutor y tutorado, entran en un ejercicio social de interacción y colaboración en pos de la mejora del texto. No solo se pretende reflexionar por medio de la lectura en voz alta y la formulación de preguntas, sino que, además, se nutre el pensamiento crítico y el proceso de retroalimentación del tutorado a través de la marcación, la elección, la interpretación y la lectura del estudiante, estrategias todas con un considerable nivel de presencia. Asimismo, estas dos últimas estrategias son más utilizadas por la
TE en las tutorías presenciales, lo que nos lleva a plantear que las tutorías virtuales debieran acercarse más a establecer la interacción y el diálogo sobre el proceso de escritura durante la sesión.

Por otro lado, parece coherente la mayor frecuencia de estrategias cognitivas de marcación, pregunta y recomendación en las tutorías virtuales, dado que al compartir el texto en la pantalla, la sesión se centra más en él y, por tanto, se marcan los errores o problemas. Asimismo, la pregunta es más necesaria al estar en línea y no tener total acceso al lenguaje paraverbal y no verbal de ambos participantes, por lo que es imperativo suplir esta falta a través de preguntas.

Llama la atención, también, que la estrategia de solución sea una de las más utilizadas. Si bien el resultado se debe a un uso elevado por parte de la TN en las tutorías presenciales, la TE la llega a emplear el doble de ocasiones en las virtuales. Esto puede dar cuenta de la dificultad que encuentran los tutores pares de establecer otro tipo de estrategias más significativas con independencia de su nivel de experticia. Al respecto, cabría abogar, en la medida de las posibilidades, por un modelo de formación continua de tutores, en donde periódicamente se revisaran las tutorías realizadas, más aún cuando el cambio de las tutorías al entorno virtual no se haya producido de forma gradual, como ha sucedido en el contexto actual de pandemia.

Asimismo, es necesario profundizar en las estrategias instructivas y motivadoras y su relación con la cortesía verbal, y cómo es esperable que las motivadoras (ánimo, empatía, identificación, interés, refuerzo y significación) estén más presentes en las sesiones virtuales, como una manera de construir un vínculo y motivar al estudiante, al no estar cara a cara. Por ejemplo, es más frecuente la sugerencia en las sesiones virtuales y esta es una estrategia más atenuada que la orientación. Como la sesión es en línea, es crucial construir el vínculo tutor-tutorado de otras maneras, mediante la identificación con el otro, interés en él, el refuerzo de su trabajo y darle sentido a la tutoría a través de la significación, 
con el fin de no perder la implicación del estudiante a pesar de que esté distanciado físicamente.

En términos de educación a distancia, algunos trabajos destacan el desarrollo de la interactividad y la colaboración como factores clave para lograr los aprendizajes deseados no solo en cuanto a la interactividad entre los sujetos, sino también del estudiante con el contenido (Hewett, 2006). Así, al observar la construcción de un vínculo con el tutorado en este estudio, por medio de un predominio de estrategias motivadoras en las sesiones virtuales, se busca implementar una interacción en donde no hay coincidencia física. Esto ha sido especialmente relevante y necesario en el contexto de pandemia en el que se han llevado a cabo las tutorías virtuales del presente estudio.

Dicho esto, cabe señalar que existen numerosas investigaciones acerca del funcionamiento de los centros de escritura y de las tutorías, pero a través de encuestas, entrevistas y grupos focales, es decir, a partir de la percepción o satisfacción de los participantes. Sin embargo, son escasos los estudios que indagan sobre las prácticas tutoriales con un enfoque ecológico e in situ, en otras palabras, que analizan qué ocurre en ellas y cómo son sus interacciones, para construir aprendizajes en torno a los procesos de escritura, especialmente en Iberoamérica, por lo que esta ha sido la principal aportación del presente trabajo. Nos parece que para construir conocimiento fidedigno sobre cómo son las tutorías de escritura, cuáles son sus aportes y cómo se pueden mejorar, es imperativo estudiar las prácticas tutoriales y, así, conformar un cuerpo robusto de conocimientos que contribuyan a apoyar y andamiar la escritura de los estudiantes y a formar tutores de escritura en educación superior.

Además, como se ha indicado, es recomendable tomar estos resultados con cautela, pues es necesario disponer de un mayor corpus de tutorías. En consecuencia, es preciso continuar con el análisis de una mayor cantidad de tutorías de más tutores noveles y expertos en los dos entornos objeto del estudio, presencial y virtual. En último término, cabe seguir interrogándose sobre cuáles son las estrategias prototípicas de una buena tutoría. De este modo, estas variables podrían tener relación con otras, en cuanto a las características del estudiante, su competencia previa y posterior en expresión escrita, y su grado de satisfacción, motivación y seguridad tras las tutorías; el objetivo de la tutoría, el género discursivo trabajado en la sesión y la fase del proceso de escritura del texto; y la formación del tutor, su grado de experticia y evolución, y la familiaridad con la disciplina del estudiante.

También consideramos necesario ahondar en el uso y las funciones de los aspectos multimodales en las tutorías virtuales y así atender a la influencia que pueden tener factores como la proyección del texto en la pantalla, el uso de la pizarra digital o del chat en las propias videoconferencias.

Por último, asimismo cabe preguntarse si las tutorías virtuales, cuyo aumento ha sido considerable durante la pandemia, puede asentarse no tanto en una alternativa aislada, cuanto en una opción útil para el acompañamiento del proceso de escritura a los estudiantes universitarios en centros de escritura en donde no eran una práctica habitual.

\section{Referencias}

Álvarez, I., Espasa, A. y Guasch, T. (2011). The value of feedback in improving collaborative writing assignments in an online learning environment. Studies in Higher Education, 37(4), 387-400. https://doi.org/ 10.1080/03075079.2010.510182

Alzate, G. M. y Peña, L. B. (2010). La tutoría entre iguales: una modalidad para el desarrollo de la escritura en la educación superior. Universitas Psychologica, 9(1), 123-138. https://doi.org/10.11144/Javeriana. upsy9-1.timd

Assarroudi, A., Heshmati Nabavi, F., Armat, M. R., Ebadi, A. y Vaismoradi, M. (2018). Directed qualitative content analysis: The description and elaboration of its underpinning methods and data analysis process. Journal of Research in Nursing, 23(1), 42-55. https://doi.org/10.1177/1744987117741667 
Bach, C. y Montané, A. (2016). Mentories de llengua per a la millora de les competències de redacció acadèmica. Revista CIDUI, (3), 1-10. https://repositori.upf. edu/handle/10230/35467

Caldwell, E., Stapleford, K. y Tinker, A. (2018). Talking academic writing: A conversation analysis of oneto-one learning development tutorials. Journal of Academic Writing, 8(2), 124-136. https://doi. org/10.18552/joaw.v8i2.464

Calle-Arango, L. (2019). La pregunta en las tutorías de los centros de escritura. Íkala, Revista de Lenguaje y Cultura, 24(1), 137-152.https://doi.org/10.17533/ udea.ikala.v24n01a07

Castellà, J. M. y Aliagas, C. (2016). La mentoría entre iguales para el aprendizaje de la escritura académica. Una experiencia en la Universidad Pompeu Fabra de Barcelona. En G. Bañales, M. Castelló y N. A. Vega (Eds.), Enseñar a leer y a escribir en la educación superior. Propuestas educativas basadas en la investigación (pp. 281-314). sM.

Castro, M. C., Hernández, L. A. y Sánchez, M. (2010). El ensayo como género académico: una aproximación a las prácticas de escritura en la universidad pública mexicana. En G. Parodi (Coord.), Alfabetización académica y profesional en el siglo XXI. Leer y escribir desde las disciplinas (pp. 49-70). Ariel/Academia Chilena de la Lengua.

Chois, P. M., Casas, A. C., López, A., Prado, D. M. y Cajas, E. Y. (2017). Percepciones sobre la tutoría entre pares en escritura académica. Magis. Revista Internacional de Investigación en Educación, 9(19), 165-184. https://doi.org/10.11144/Javeriana.m9-19.ptpe

Cooper, M. M. (1994). Really useful knowledge: A cultural studies agenda for writing centers. The Writing Center Journal, 14(2), 97-111. https://www.jstor.org/ stable/43441948

Cromley, J. G. y Azevedo, R. (2005). What do reading tutors do? A naturalistic study of more and less experienced tutors in reading. Discourse Processes, 40(2), 83-113. https://doi.org/10.1207/s15326950dp4002_1

Dinitz, S. y Kiedaisch, J. (2003). Creating theory: Moving tutors to the center. The Writing Center Journal, 23(2), 63-76. http://www.pitt.edu/ writecen/ WCJ23.2_Dinitz_Kiedaisch.pdf

Eastmond, L. (2019). Scaffolding in the center: Training tutors to facilitate learning interactions with L2 Writers [Tesis de doctorado, Arizona State University]. Repositorio Arizona State University. https://core. ac.uk/download/pdf/286400723.pdf
Errázuriz, M. C. (2016). The academic writing development in the initial teacher training: Tutoring in a writing centre as a strategy for the modelling of students. The International Journal of Literacies, 23(3), 27-43. https://repositorio.uc.cl/handle/11534/38761

Errázuriz, M. C. (2017). Las tutorías de un centro de escritura como dispositivo de modelamiento de estudiantes de programas de formación inicial docente. En L. Natale y D. Stagnaro (Eds.), Alfabetización académica: un camino para la inclusión en el nivel superior (pp. 103-131). UNGs.

Errázuriz, M. C. (2019). Desempeño escrito de estudiantes de programas de formación inicial docente: ¿cómo es la calidad del proceso de escritura de sus ensayos? Lengua y Habla, 23, 224-242. http:// erevistas.saber.ula.ve/index.php/lenguayhabla/ article/view/15670/21921926771

Ezcurra, A. M. (2013). Igualdad en educación superior. Un desafio mundial. Universidad Nacional de General Sarmiento.

García-Arroyo, M. y Quintana, H. (2016). La capacitación profesional de los tutores en los centros de escritura. En G. Bañales, M. Castelló y N. A. Vega (Eds.), Enseñar a leer y a escribir en la educación superior. Propuestas educativas basadas en la investigación (pp. 364-389). sM.

Harrington, K., O’Neill, P. y Bakhshi, S. (2007). Writing mentors and the writing centre: Producing integrated disciplinary writers. Investigations in university teaching and learning, 4(2), 26-32. http://repository. londonmet.ac.uk/241/1/InvestigationsInUniversityTeachingAndLearning\%20v4n2\%20p26-32.pdf

Hewett, B. (2006). Synchronous online conferencebased instruction: A study of whiteboard interactions and student writing. Computers \& Composition, 23(1), 4-31. https://doi.org/10.1016/j. compcom.2005.12.004

Holtz, E. V. (2014). Mode, method, and medium: The affordance of online tutorials in the writing center. Honors Scholar Theses, 357. https://opencommons. uconn.edu/srhonors_theses/357

Jones, R., Garralda, A., Li, D. y Lock, G. (2006). Interactional dynamics in on-line and face-to-face peer-tutoring sessions for second language writers. Journal of Second Language Writing, 15(1), 1-23. https://doi. org/10.1016/j.jslw.2005.12.001

Kirchhoff, L. (2016). Motivation in the writing centre: A peer tutor's experience. Journal of Academic Writing, 6(1), 31-40. https://doi.org/10.18552/joaw.v6i1.282 
Mackiewicz, J. (2004). The effects of tutor expertise in engineering writing: A linguistic analysis of writing tutors' comments. IEEE Transactions on Professional Communication, 47(4), 316-328. https://doi. org/10.1109/TPC.2004.840485

Mackiewicz, J. (2005). Hinting at what they mean: Indirect suggestions in writing tutors' interactions with engineering students. IEEE Transactions on Professional Communication, 48(4), 365-376. https://doi. org/10.1109/TPC.2005.859727

Mackiewicz, J. y Thompson, I. (2014). Instruction, cognitive scaffolding, and motivational scaffolding in writing center tutoring. Composition Studies, 42(1), 54-78. https://doi.org/10.4324/9781351272643

Mackiewicz, J. y Thompson, I. (2018). Talk about writing. The tutoring strategies of experienced writing center tutors. Routledge. https://doi. org/10.4324/9781351272643-9

Martínez, P., Pérez, J. y Martínez, M. (2016). Las Tics y el entorno virtual para la tutoría universitaria. Educación XXI, 19(1), 287-310. https://doi.org/10.5944/ educxx1.13942

Molina-Natera, V. (2019). El discurso pedagógico en las tutorías de escritura. Develando elementos de una práctica educativa. Revista Mexicana de Investigación Educativa, 24(80), 125-148. http://www.scielo.org.mx/pdf/ rmie/v24n80/1405-6666-rmie-24-80-125.pdf

Monty, R. W. (2013). Theoretical communities of praxis: The university writing center as cultural contact zone (Tesis de Doctorado, The University of Texas at El Paso). Repositorio The University of Texas at El Paso. https:// scholarworks.utep.edu/open_etd/1889/

Muñoz, C. y Valenzuela, J. (2015). Características psicométricas de una rúbrica para evaluar expresión escrita a nivel universitario. Formación Universitaria, 8(6), 75-84. http://dx.doi.org/10.4067/ S0718-50062015000600010

Natale, L. (2016). Las devoluciones escritas del profesor universitario. En L. Natale y D. Stagnaro (Eds.), La lectura y la escritura en las disciplinas. Lineamientos para su enseñanza (pp. 167-200). UNGS.

Natale, L. y Stagnaro, D. (Eds.). (2017). Alfabetización académica: un camino para la inclusión en el nivel superior. UNGS.

North, S. M. (1982). Training tutors to talk about writing. College Composition and Communication 33(4), 434-441. https://doi.org/10.2307/357958

Núñez, J. A. (2020). Las tutorías de escritura académica: estrategias didácticas de una tutora novel.
Enunciación, 25(2), 176-190. https://doi. org/10.14483/22486798.16563

Núñez, J. A. y García, M. J. (2018). Escribir en las universidades a distancia: formación y dificultades de los estudiantes. Revista Tecnología, Ciencia y Educación, (11), 47-60. https://doi.org/10.51302/ tce. 2018.222

Núñez, J. A. y Errázuriz, M. C. (2020). Panoramas de la alfabetización académica en el ámbito iberoamericano: aportes para la calidad de la educación superior. Tendencias Pedagógicas, 36, 1-8. https:// doi.org/10.15366/tp2020.36.01

Parodi, G. (Ed.). (2008). Géneros académicos y géneros profesionales. Accesos discursivos para saber y hacer. Universitarias de Valparaíso.

Piaget, J. (1970). The science of education and the psychology of the child. New York: Orion Press.

Roldán, C. A. y Arenas, K. A. (2016). Características de las tutorías del Centro de Lectura y Escritura de la Universidad Autónoma de Occidente: ¿qué muestran los registros de atención? Revista Grafia,13(1), 100-114. https://doi. org/10.26564/16926250.658

Ryan, L. y Zimmerelli, L. (2006). The Bedford guide for writing tutors. Bedford/St.Martin's Press.

Scardamalia, M. y Bereiter, C. (1992). Dos modelos explicativos de los procesos de composición escrita. Infancia y aprendizaje, 15(58), 43-64. https://doi.org/10.10 80/02103702.1992.10822332

Skagen, T., Torras, M., Kavli, S. M., Mikki, S., Hafstad, S. y Hunskår, I. (2009). Pedagogical considerations in developing an online tutorial in information literacy. Communications in Information Literacy, 2(2), 84-98. https://doi.org/10.15760/ comminfolit.2009.2.2.60

Sun, Y., Correa, M., Zapata, A. y Carrasco D. (2011). Resultados: qué dice la evaluación docente acerca de la enseñanza en Chile. En J. Manzi, R. González y Y. Sun (Eds.), La evaluación docente en Chile (pp. 91135). MIDE UC/Ediciones UC.

Thaiss, C., Bräuer, G., Carlino, P., Ganobcsik-Williams, L. y Sinha, A. (Eds.). (2012). Writing programs worldwide: Profiles of academic writing in many places. The WAC Clearinghouse \& Parlor Press. https://doi. org/10.37514/PER-B.2012.0346

Thompson, I. (2009). Scaffolding in the writing center: A microanalysis of an experienced tutor's verbal 
and nonverbal tutoring strategies. Written Communication, 26(4), 417-453. https://doi. org/10.1177/0741088309342364

Unesco. (2020, marzo). La educación en América Latina y el Caribe ante la COVID-19. https://es.unesco.org/ fieldoffice/santiago/covid-19-education-alc

Van Eemeren, F. H. (2001). Crucial concepts in argumentation theory. Amsterdam University Press.
Vygotsky, L. (1978). Mind in society. The development of higher psychological processes. Harvard University Press.

Yergeau, M., Wozniak, K. y Vandenberg, P. (2008). Expanding the space of $\mathrm{f} 2 \mathrm{f}$ : Writing centers and audio-visual-textual conferencing. Kairos. $A$ Journal of Rhetoric, Technology, and Pedagogy, 13(1). http://www.technorhetoric.net/13.1/index.html

Cómo citar este artículo: Núñez-Cortés, J. A., Errázuriz, M. C., Neubauer Esteban, A. y Parada, C. (2021). Las tutorías de escritura académica presenciales y virtuales: ¿qué podemos aprender sobre sus estrategias didácticas? Íkala, Revista de Lenguaje y Cultura, 26(3), 643-660. https://doi.org/10.17533/ udea.ikala.v26n3a10 